\title{
Cost-effectiveness of docetaxel in high-volume hormone-sensitive metastatic prostate cancer
}

Jaclyn Beca ${ }^{1}$; Habeeb Majeed ${ }^{2}$; Kelvin K.W. Chan ${ }^{3}$; Sebastian J. Hotte ${ }^{4}$; Andrew Loblaw ${ }^{3}$; Jeffrey S. Hoch ${ }^{5}$

${ }^{1}$ Cancer Care Ontario, Canada; ${ }^{2}$ University Health Network, Toronto, ON, Canada; ${ }^{3}$ Sunnybrook Hospital, Toronto, ON, Canada; ${ }^{4}$ Hamilton Heath Sciences Centre, Hamilton, ON, Canada; ${ }^{5}$ UC Davis, Davis, California, United States

Cite as: Can Urol Assoc J 2019 April 26; Epub ahead of print. http://dx.doi.org/10.5489/cuaj.5889

Published online April 26, 2019

$* * *$

\section{Abstract}

Introduction: Three pivotal trials have considered the addition of docetaxel (D) chemotherapy to conventional androgen-deprivation therapy (ADT) for the treatment of metastatic hormonesensitive prostate cancer (HSPC). While an initial small trial was inconclusive, two larger trials demonstrated significant clinical benefit, including pronounced survival benefits (added 17 months) among patients with high-volume metastatic disease. Given the evolving clinical evidence, the cost-effectiveness of this approach warrants exploration.

Methods: The cost-effectiveness of six cycles of ADT+D compared to ADT alone to treat patients with high-volume metastatic HSPC was assessed from a Canadian public payer perspective. We included three health states: HSPC, metastatic castration-resistant prostate cancer (CRPC), and death. Survival data were obtained from the CHAARTED trial, which reported outcomes specifically for high-volume disease. We used Ontario costs data and utilities from the literature.

Results: In the base case analysis, ADT+D cost an additional \$25 757 and produced an extra 1.06 quality-adjusted life years (QALYs), resulting in an incremental cost-effectiveness ratio (ICER) of \$24 226/QALY gained. Results from one-way sensitivity analysis across wide ranges of estimates and a range of scenarios, including an alternate model structure, produced ICERs below \$35 000/QALY gained in all cases.

Conclusions: The use of D with ADT in high-volume metastatic HSPC appears to be an economically attractive treatment approach. The findings were consistent with other studies and robust in sensitivity analysis across a variety of scenarios. 


\section{Introduction}

Prostate cancer is the most commonly diagnosed cancer among males, representing $23.9 \%$ of all cancers diagnosed among men, with an annual incidence of 24,000 in Canada ${ }^{1}$ and over 220,800 in the United States. ${ }^{2}$ With an estimated 307,000 deaths in 2012, it is the fifth leading cause of cancer-related death among men worldwide. ${ }^{3}$

The proliferation of prostate cancer is largely mediated through the androgen receptor pathway; therefore, by reducing the level of circulating androgen through androgen deprivation therapy (ADT) one can impede further proliferation of disease. ${ }^{4}$ However, in using ADT alone, patients will eventually develop androgen resistance, becoming castration-resistant. ${ }^{5}$

For metastatic castration-resistant prostate cancer (CRPC), the results of the TAX $327^{6}$ and SWOG-9916 ${ }^{7}$ trials using docetaxel showed overall survival benefit relative to mitoxantrone. These studies raised the question as to whether men with hormone-sensitive prostate cancer (HSPC) could benefit from adding this chemotherapy to improve outcomes.

Three pivotal trials have looked at the addition of docetaxel to first-line ADT for the treatment of metastatic HSPC. In the first reported trial, GETUG-AFU15, 385 men with metastatic HSPC were randomized to ADT alone or ADT plus docetaxel $75 \mathrm{mg} / \mathrm{m}^{2}$ every three weeks for up to nine cycles. ${ }^{8}$ At median follow-up of 84 months, there was improvement in biochemical progression-free survival (median 22.9 versus 12.9 months, $\mathrm{HR}=0.67,95 \% \mathrm{CI}=0.54$ 0.84). There was no statistically significant increase in overall survival (OS); however, in an unplanned post-hoc analysis, there was a $20 \%$ reduction in risk of death in the high-volume disease group that failed to reach statistical significance. ${ }^{9}$ In the CHAARTED trial (E3805), ${ }^{10}$ 790 men with treatment-naïve metastatic HSPC were randomized to ADT alone or ADT plus six cycles of docetaxel at $75 \mathrm{mg} / \mathrm{m}^{2}$ every three weeks. OS was significantly increased with ADT plus docetaxel compared to ADT alone (median 57.6 versus 44 months, $\mathrm{HR}=0.61,95 \% \mathrm{CI}=0.47$ 0.80 ). This trend was most pronounced among patients with high-volume disease, defined by visceral metastases and/or four or more bone metastases, with median OS of 49.2 versus 32.2 months favouring the docetaxel group. The median time to biochemical, symptomatic, or radiographic progression was also significantly longer with ADT plus docetaxel (20 versus 12 months, $\mathrm{HR}=0.61,95 \% \mathrm{CI}=0.52-0.72$ ). Long-term results after 53.7 months median follow-up were consistent with initial reports, including significant increase in OS for ADT plus docetaxel of 51.2 versus 34.4 months in the high-volume subgroup ( $\mathrm{HR}=0.63,95 \% \mathrm{CI}=0.50-0.79){ }^{11,12}$ Finally, the STAMPEDE trial ${ }^{13}$ randomized 2,962 men to one of four different treatment regimens, including long-term ADT or ADT plus docetaxel $75 \mathrm{mg} / \mathrm{m}^{2}$ every three weeks for six cycles. The addition of docetaxel to ADT improved OS (median 81 versus 71 months, HR=0.78, $95 \% \mathrm{CI}=0.66-0.93$ ) and failure-free survival (median 37 versus 20 months, $\mathrm{HR}=0.61$, $95 \% \mathrm{CI}=0.53-0.70$ ) compared to ADT alone. This trial included $24 \%$ non-metastatic patients, and the OS improvement appeared to be enhanced for patients with metastases (HR $=0.76$, $95 \% \mathrm{CI}=0.62-0.92)$. However, unlike the other two trials, the proportion and outcomes for 
patients with high-volume disease were not reported. Meta-analyses of these trials ${ }^{14-16}$ have consistently demonstrated that addition of docetaxel is associated with OS benefit, particularly among those with high-volume metastatic disease.

In addition to the survival benefit, the impact of docetaxel on quality of life (QOL) is an important consideration. Though adverse events were higher with docetaxel in the CHAARTED trial, ${ }^{10}$ in the long run, at 12 months from time of treatment, QOL was better in the docetaxel and ADT arm. ${ }^{17}$ The implication is that ADT plus docetaxel may be a favourable intervention that provides not only a survival benefit, but also preserves better QOL for metastatic HSPC than ADT alone.

These studies have practice-changing implications and the transition of docetaxel earlier into the hormone-sensitive space needs to be formally evaluated from a cost-effectiveness perspective. The objective of this study was to evaluate, from a Canadian public payer perspective, the cost-effectiveness of 6 cycles of docetaxel plus ADT compared to ADT alone to treat patients with high-volume metastatic HSPC. The results were used to inform decisionmaking for the Ontario public health care system.

\section{Methods}

\section{Model structure}

An economic model was constructed to conduct a cost-effectiveness analysis and a cost-utility analysis following the guidelines outlined by CADTH. ${ }^{18} \mathrm{~A}$ partitioned survival model was developed for high-volume metastatic prostate cancer with three health states: HSPC, CRPC, and death (Figure 1). A partitioned survival model uses area under the curve to determine mean time spent in each state. This approach was chosen because time-to-event survival curves for time to CRPC and OS endpoints were relatively complete for both treatment groups, thus providing good estimation of the time spent in each health state with little need for extrapolation.

Our model assumed all patients begin in the HSPC state, are chemotherapy naïve for metastatic disease, eligible to receive ADT and have patient characteristics in line with the CHAARTED trial, which was the most recent trial data available at the time of initiation of this analysis and largest reported group of high-volume patients. ${ }^{10}$ The time horizon used for the analysis was 15 years based on clinical input. This analysis was conducted in Ontario from the government perspective. Outcomes and costs were discounted at $1.5 \%$ per year. ${ }^{18}$

\section{Comparators}

We compared 6 cycles of docetaxel at $75 \mathrm{mg} / \mathrm{m}^{2}$ given every 3 weeks plus ADT (ADT + D) to ADT alone in metastatic HSPC. In accordance with the trial protocol, we considered ADT as a class of therapy using leutinizing hormone releasing hormone (LHRH) therapy (agonists or antagonists). 


\section{Clinical data}

In the base case analysis, the survival curves from the high-volume subgroup in the CHAARTED study were used to estimate time to CRPC and OS for each treatment group. The Kaplan Meier survival curves from the latest follow-up of 53.7 months ${ }^{11,12}$ were digitized using Engauge digitization software and patient-level data were estimated. ${ }^{19}$ Survival curves were extrapolated using independently fit parametric curves for each treatment arm according to best practice, ${ }^{20}$ displayed in Figure 2 and parameters listed in Table 1.

In sensitivity analyses, we tested alternative extrapolation approaches. In one scenario, a hazard ratio from meta-analysis was applied to the ADT OS curve to explore uncertainty in the relative benefit. We also explored results using alternate parametric distributions (exponential, Weibull, lognormal, log-logistic, Gompertz and generalized gamma).

A Markov model was also developed to model transitions from HSPC to CRPC and CRPC to death separately. This was conducted as a scenario only because there were no direct data to estimate mortality transition probabilities. The transition probability from HSPC to CRPC was calculated from time-to-CRPC curves, and risk of death from HSPC was assumed to be equal to age-related mortality, given the asymptomatic nature of the disease. ${ }^{21}$ Risk of death from CRPC in each arm was estimated by calibrating to the median OS for each treatment group from the trial. ${ }^{11}$ When assuming equal risks of death from CRPC regardless of initial treatment group, the average of the estimated risks from each arm were used for both arms. Transition probabilities for the scenario analyses are also summarized in Table 1.

\section{Costs}

Costs are reported in 2017 Canadian dollars (Table 1). While the costs for LHRH therapy differ depending on drug and formulation, on average the monthly costs of these medications are approximately $\$ 300-400 .^{22}$ We estimated the costs using leuprolide (Lupron Depot) 22.5mg 3month formulation injection kit. We also added management costs, including nursing time, administrative support, clerical work, and pharmacy time for preparation (Cancer Care Ontario payment model data) for patients on intravenous (IV) or on non-IV therapy and we assumed patients would visit the oncology clinic monthly.

Docetaxel costs were estimated using the trial dosing, unit cost of docetaxel at the time of the analysis $\left(\$ 0.54 / \mathrm{mg}\right.$ ) (Cancer Care Ontario price list) and BSA $1.8 \mathrm{mg} / \mathrm{m}^{2}$, without dose modifications for up to 6 cycles, as long as patients remained in the HSPC state. In the CHAARTED trial, $74 \%$ of the patients received all 6 cycles of docetaxel without dose modifications or delays in therapy; patients received an average 5.65 cycles of docetaxel. ${ }^{10}$

Treatment for CRPC includes abiraterone, docetaxel, as well as enzalutamide or cabazitaxel. According to clinical opinion, patients who use docetaxel in the HSPC setting could still potentially benefit from docetaxel in CRPC. Thus, we assumed no differences in the treatment pathway beyond the development of castrate resistance i.e., both groups could receive the same basket of therapies, including retreatment with docetaxel. We assumed an average 
monthly cost for treatment and management in the metastatic CRPC state as one progressive health state combined. To be conservative, we estimated the average monthly cost based on first line abiraterone $(\$ 3,602)^{23}$ plus (non-IV) disease management costs to encompass regular clinic visits and nursing support. Abiraterone and enzalutamide are similarly priced, ${ }^{23}$ most widely used for metastatic CRPC, and were the most commonly used of the available therapies after progression in the CHAARTED trial. ${ }^{10}$ Thus, we represented costs for the CRPC state using monthly costs for these two expensive therapies. We assumed all other costs are the same between treatment groups (e.g., physician visits). In this model, it is assumed patients are always on ADT or CRPC therapies, and longer survival produces added costs.

\section{Adverse events}

In the CHAARTED trial, $6 \%$ of patients on ADT + docetaxel had febrile neutropenia and 2\% had grade $3 / 4$ neutropenia with infection. ${ }^{10}$ We included the cost of hospitalization to treat febrile neutropenia ${ }^{24}$ (adjusted to 2017 dollars) ${ }^{25}$ for all $8 \%$ of patients. We did not include other adverse events as all other grade $3 / 4$ adverse events occurred in less than $4 \%$ of patients in the CHAARTED trial. We assumed no differences in adverse event rates from CRPC therapies.

\section{Utilities}

A literature review of previous economic analyses was conducted to capture estimates of health state utility (Table 1). Patients with metastatic HSPC were assumed to have a utility value of 0.9 , consistent with estimates in previous economic evaluations for patients with asymptomatic metastatic prostate cancer. ${ }^{26-28}$ Patients with metastatic CRPC were assigned a utility value of 0.77 , consistent with estimates used in an evaluation of abiraterone for patients with metastatic CRPC. $^{29}$

\section{Analysis}

Base case

We conducted probabilistic analysis to account for uncertainty in parameters. Beta distributions were used for utilities and probabilities, gamma distributions for costs and lognormal distributions for the utility decrement and median durations. For the correlated uncertainty in the extrapolation parameters, we used normal distributions and the Cholesky decomposition. For the probabilistic analysis, 5,000 simulations were conducted. The average costs and effects for each treatment group were used to estimate incremental costs, incremental effects, and the incremental cost-effectiveness ratio (ICER).

Sensitivity analysis

One-way sensitivity analysis (OWSA) was performed on the survival estimates, costs, and utilities used in the model. We tested scenarios with an estimate of relative treatment benefit 
from meta-analysis, alternate utility values, alternate distributions for survival extrapolation and we explored structural model assumptions using a Markov model.

\section{Results}

In the base case probabilistic analysis, ADT + docetaxel has an incremental cost of $\$ 25,757$ and produced an extra 1.063 QALYs, resulting in an ICER of \$24,226/QALY gained (Table 2). Longer survival in both health states were each associated with higher costs for the ADT + docetaxel arm. A scatterplot of the probabilistic analysis showed all iterations produced added benefits, and nearly all also resulted in added costs (Figure 3a). Over 99\% of iterations were considered cost-effective at a willingness-to-pay threshold of \$50,000/QALY gained (Figure 3b).

We conducted a series of OWSA to evaluate each model parameter. Overall, the model was most sensitive to the cost of treatment and management in the CRPC health state, with lower costs improving the cost-effectiveness of the initial intervention, as well as the survival distributions chosen for CRPC and OS curves. However, no scenario nor parameter change increased the ICER by more than $\$ 10,000 / Q A L Y$ gained, i.e., all scenarios and OWSA produced ICERS below $\$ 35,000 /$ QALY gained (Figure 4).

As the impact of the use of initial docetaxel in metastatic HSPC on the outcomes from the downstream CRPC period is uncertain, we also explored uncertainty in the time horizon and the model structure. Using the observed survival outcomes only, i.e., a time horizon of 7.5 years, survival was truncated early, resulting in 0.93 discounted LYs, and the ICER was lowered (\$17,056/QALY gained) due to lower CRPC costs. Using the hazard ratio from the meta-analysis of trials ${ }^{14}$ also produced a lower ICER of \$16,966/QALY gained.

A Markov model was used to model the transition from metastatic HSPC to CRPC and CRPC to death separately. As no data are available to inform the effect of initial metastatic HSPC therapy on the risks after developing CRPC, two assumptions were possible. First, assuming no differences in risk of death from CRPC between treatment strategies resulted a lower ICER than the base case (\$3,985/QALY gained). This scenario predicted 0.80 discounted LYs, smaller than the survival difference observed in the trial data between the strategies, which also reduced the difference in extra cost arising during the CRPC health state (additional \$2,766 discounted costs). To better align the results of the Markov model with the observed data, we calibrated the probability of death from the CRPC health state to the median OS in each arm, thereby assuming a lower risk of death from CRPC from early docetaxel that produces additional benefit in the CRPC period. This produced a larger OS benefit (1.39 discounted LYs) and slightly larger ICER of \$27,354/QALY gained, due to the added costs in the CRPC period (additional \$31,392 discounted costs).

\section{Discussion}

This study, to our knowledge, is the first to attempt to quantify the cost-effectiveness of the addition of docetaxel to ADT in metastatic HSPC from a North American healthcare system 
perspective. Our study demonstrates that the clinical benefits achieved for patients with high volume metastatic HSPC also appear to be economically attractive and viable from a public payer approach, with an ICER below commonly accepted willingness-to-pay thresholds of $\$ 50,000$ - \$100,000/QALY gained. Though this study was conducted using Ontario inputs, treatment patterns and pricing are expected to be similar across Canada, particularly in light of joint negotiations and implementation discussions for new and expensive treatment options through the pan-Canadian Pharmaceutical Alliance (pCPA); thus, we expect these findings are also relevant to other Canadian jurisdictions.

In comparing our findings to other studies, one study in China ${ }^{30}$ found that adding docetaxel to ADT among patients with high-volume disease increased cost and effectiveness by US\$14,628 and 0.69 QALYs, respectively, producing an ICER of US\$21,200/QALY gained. One other study conducted in Brazil reported the combination of docetaxel with ADT produced an additional 0.70 QALYs at added costs in high-volume metastatic disease, resulting in an ICER of US\$8,417/QALY gained. ${ }^{31}$ Both of these studies were conducted using the initial CHAARTED trial data from median follow-up of 28.9 months. ${ }^{10}$

When evaluating the cost effectiveness of early docetaxel in metastatic HSPC, it is also useful to put the results in context relative to other interventions in metastatic prostate cancer. The use of docetaxel for metastatic CRPC was associated with an ICER of £28,000$£ 33,000$ /QALY gained relative to mitoxantrone when it was initially funded in this setting (and prior to becoming generic). ${ }^{32}$ Abiraterone, in three separate cost effectiveness analyses ${ }^{33-35}$ in metastatic CRPC was found to have an ICER between $\$ 94,000 /$ QALY $^{33}$ and \$389,000/QALY gained. ${ }^{34}$ Enzalutamide and cabazitaxel in metastatic CRPC had ICERs of \$154,300/QALY gained $^{35}$ and $\$ 163,200 /$ QALY gained ${ }^{35}$ relative to placebo, respectively. In comparing the aforementioned treatment options in metastatic prostate cancer, one can see how older therapeutic drugs that have come off patent can be very economically attractive. From a research development perspective, there may be potential of extracting further cost-effective treatment options by taking established, proven, and inexpensive drugs and applying them in new clinical settings rather than simply searching for new molecule discovery. This is also particularly relevant in light of the findings of the LATITUDE-3 trial studying the addition of abiraterone to ADT for high-risk metastatic HSPC, which appeared to produce similar clinical benefits as docetaxel (OS HR $=0.62$ compared to ADT alone) ${ }^{36}$ but at much higher cost; in the study, patients were treated for a median duration of 24 months at a cost $\$ 3,600$ per month, ${ }^{23}$ an over 200 -fold increase in costs for the average course of abiraterone compared to docetaxel to achieve these similar benefits. A recent network meta-analysis of five trials with high-risk, metastatic HSPC found no OS differences between abiraterone and docetaxel in this setting, (OS HR $=0.84$, $95 \% \mathrm{CI}=0.67-1.06) .{ }^{37}$ While it would appear from indirect comparison of OS that docetaxel provides comparable benefits in a more efficient manner, the cost-effectiveness of abiraterone compared to docetaxel in the HSPC setting is another relevant policy question for future funding 
consideration. Further exploration of treatments in this setting may be of particular interest for those ineligible for chemotherapy and in future as we anticipate price reductions for abiraterone from generic entrants. From an evaluation perspective, our model can be used in future studies to formally evaluate abiraterone informed by future direct evidence or a network meta-analysis and indirect comparison of all the relevant survival and adverse event endpoints, along with evaluation of other additional therapies that may enter this treatment space in future.

Our analysis had some limitations. As stated above, we did not include all adverse event costs or impact of all specific adverse events on QOL since the frequency of these events was relatively small in the original CHAARTED trial. ${ }^{11,12}$ Given the treatment is a short-term add-on therapy in relatively asymptomatic patients fit for ADT, this was unlikely to impact our results; moreover, we incorporated a large utility decrement for the treatment period with docetaxel to account for negative QOL impact of IV chemotherapy. Our analysis, therefore, focused only on the costliest and most frequent of adverse events that could impact the cost-effectiveness of the intervention. We simplified the costing and utilities into two disease states, but tested costs and utilities for each using a wide range in sensitivity analysis in both Markov and partitioned survival model structures. Our model does not explicitly model and test treatment sequences in the CRPC setting, though it makes a conservative assumption that patients receive expensive antiandrogens for the duration of CRPC until death. To conduct our sensitivity analysis with a Markov model, there were no data available to directly estimate death from CRPC specific to patients with high-volume disease, either from the trial or in the literature. On the other hand, the observed data captured near-complete follow-up for the cohort (survival $20 \%$ and $10 \%$ in the $\mathrm{ADT}+\mathrm{D}$ and $\mathrm{ADT}$ arms, respectively), meaning minimal extrapolation was required, reducing the uncertainty typically associated with the partitioned survival model structure. Finally, we did not evaluate the impact of the addition of docetaxel to locally advanced (M0), non-metastatic HSPC as assessed in the subsequent STAMPEDE clinical trial. ${ }^{13}$ We felt that the evidence supports the addition of docetaxel to ADT only for those with high-volume metastatic disease, as a recent meta-analysis demonstrated that despite an impact on failure-free survival ( $\mathrm{HR}=0.70$; $95 \% \mathrm{CI}=0.61-0.81 ; \mathrm{p}<0.0001)$ no OS benefit was shown among men with locally advanced, nonmetastatic disease $(\mathrm{HR}=0.87 ; 95 \% \mathrm{CI}=0.69-1.09 ; \mathrm{p}=0.218) .{ }^{15}$

In conclusion, the use of docetaxel with ADT in metastatic HSPC appears to be an economically attractive treatment approach that provides large clinical benefits for a targeted group of patients. The incremental cost-effectiveness ratio for this intervention was less than \$25,000/QALY gained in the base case, the findings were similar to those of other studies and robust to a variety of scenarios. 


\section{References}

1. Canadian Cancer Society's Advisory Committee on Cancer Statistics. Canadian Cancer Statistics 2016. Toronto ON, 2016 doi:0835-2976.

2. Siegel RL, Miller KD, Jemal A. Cancer statistics, 2015. CA Cancer J Clin 2015;65:5-29.

3. Ferlay J, Soerjomataram I, Dikshit R, et al. Cancer incidence and mortality worldwide: Sources, methods and major patterns in GLOBOCAN 2012. Int J Cancer 2015;136:E359-86.

4. Sharifi N, Gulley JL, Dahut WL. Androgen deprivation therapy for prostate cancer. JAMA 2005;294:238-44.

5. Sharifi N, Dahut WL, Steinberg SM, et al. A retrospective study of the time to clinical endpoints for advanced prostate cancer. BJU Int 2005;96:985-89.

6. Berthold DR, Pond GR, Soban F, de Wit R, Eisenberger M, Tannock IF. Docetaxel plus prednisone or mitoxantrone plus prednisone for advanced prostate cancer: updated survival in the TAX 327 study. J Clin Oncol 2008;26:242-45.

7. Petrylak DP, Tangen CM, Hussain MHA, et al. Docetaxel and estramustine compared with mitoxantrone and prednisone for advanced refractory prostate cancer. $N$ Engl $J$ Med 2004;351:1513-20.

8. Gravis G, Fizazi K, Joly F, et al. Androgen-deprivation therapy alone or with docetaxel in non-castrate metastatic prostate cancer (GETUG-AFU 15): a randomised, open-label, phase 3 trial. Lancet Oncol 2013;14:149-58.

9. Gravis G, Boher J-M, Joly F, et al. Androgen Deprivation Therapy (ADT) Plus Docetaxel Versus ADT Alone in Metastatic Non castrate Prostate Cancer: Impact of Metastatic Burden and Long-term Survival Analysis of the Randomized Phase 3 GETUG-AFU15 Trial. Eur Urol 2016;70:256-62.

10. Sweeney CJ, Chen Y-H, Carducci M, et al. Chemohormonal Therapy in Metastatic Hormone-Sensitive Prostate Cancer. N Engl J Med 2015;373:737-46.

11. Sweeney C, Chen Y-H, Liu G, et al. Long term efficacy and QOL data of chemohormonal therapy (C-HT) in low and high volume hormone naïve metastatic prostate cancer (PrCa): E3805 CHAARTED trial. Ann Oncol 2016;27. doi:10.1093/annonc/mdw372.04.

12. Kyriakopoulos CE, Chen Y-H, Carducci MA, et al. Chemohormonal Therapy in Metastatic Hormone-Sensitive Prostate Cancer: Long-Term Survival Analysis of the Randomized Phase III E3805 CHAARTED Trial. J Clin Oncol 2018;36:1080-87.

13. James ND, Sydes MR, Clarke NW, et al. Addition of docetaxel, zoledronic acid, or both to first-line long-term hormone therapy in prostate cancer (STAMPEDE): survival results from an adaptive, multiarm, multistage, platform randomised controlled trial. Lancet 2016;387:1163-77.

14. Tucci M, Bertaglia V, Vignani F, et al. Addition of Docetaxel to Androgen Deprivation Therapy for Patients with Hormone-sensitive Metastatic Prostate Cancer: A Systematic Review and Meta-analysis. Eur Urol 2016;69:563-73.

15. Vale CL, Burdett S, Rydzewska LHM, et al. Addition of docetaxel or bisphosphonates to standard of care in men with localised or metastatic, hormone-sensitive prostate cancer: a systematic review and meta-analyses of aggregate data. Lancet Oncol 2016;17:243-56. 
16. Botrel TEA, Clark O, Lima Pompeo AC, et al. Efficacy and Safety of Combined Androgen Deprivation Therapy (ADT) and Docetaxel Compared with ADT Alone for Metastatic Hormone-Naive Prostate Cancer: A Systematic Review and Meta-Analysis. PLoS One 2016;11:e0157660.

17. Patrick-Miller LJ, Chen Y-H, Carducci MA, et al. Quality of life (QOL) analysis from CHAARTED: Chemohormonal androgen ablation randomized trial in prostate cancer (E3805). J Clin Oncol 2016;34:5004-5004.

18. Guidelines for the Economic Evaluation of Health Technologies: Canada 4th Edition. Ottawa, 2017URL https://www.cadth.ca/sites/default/files/pdf/guidelines_for_the_economic_evaluation_of_ health_technologies_canada_4th_ed.pdf Accessed 8 November 2018.

19. Guyot P, Ades a E, Ouwens MJNM, Welton NJ. Enhanced secondary analysis of survival data: reconstructing the data from published Kaplan-Meier survival curves. BMC Med Res Methodol 2012;12:9.

20. Latimer NR. Survival analysis for economic evaluations alongside clinical trials-extrapolation with patient-level data: inconsistencies, limitations, and a practical guide. Med Decis Making 2013;33:743-54.

21. Life tables, Canada, provinces and territories, catalogue no. 84-537-X. URL https://www150.statcan.gc.ca/n1/pub/84-537-x/2018002/xls/2014-2016_Tbl-eng.xlsx.

22. Ontario Drug Benefit Formulary. URL https://www.formulary.health.gov.on.ca/formulary/ Accessed 8 November 2018.

23. pan-Canadian Oncology Drug Review Final Economic Guidance Report Enzalutamide (Xtandi) for Metastatic Castration-Resistant Prostate Cancer. 2013URL https://www.cadth.ca/sites/default/files/pcodr/pcodr-xtandi-mcrpc-fn-egr.pdf Accessed 8 November 2018.

24. Lathia N, Mittmann N, Deangelis C, Pharm D, Knowles S. Evaluation of Direct Medical Costs of Hospitalization for Febrile Neutropenia. 2010;742-48.

25. 25 Table 18-10-0005-01 (formerly CANSIM 326-0021) Consumer Price Index, annual average, not seasonally adjusted. URL https://www150.statcan.gc.ca/t1/tbl1/en/tv.action?pid=1810000501.

26. Bayoumi AM, Brown AD, Garber AM. Cost-effectiveness of androgen suppression therapies in advanced prostate cancer. J Natl Cancer Inst 2000;92:1731-39.

27. Lee D, Porter J, Gladwell D, Brereton N, Nielsen SK. A cost-utility analysis of degarelix in the treatment of advanced hormone-dependent prostate cancer in the United Kingdom. J Med Econ 2014;17:233-47.

28. Hatoum HT, Crawford ED, Nielsen SK, Lin S-J, Marshall DC. Cost-effectiveness analysis comparing degarelix with leuprolide in hormonal therapy for patients with locally advanced prostate cancer. Expert Rev Pharmacoecon Outcomes Res 2013;13:26170.

29. Economic evaluation of Abiraterone Acetate $\left(Z y t i g a{ }^{\circledR}\right)$ for the treatment of patients with metastatic castration-resistant prostate cancer (mCRPC) who have received prior docetaxel-based chemotherapy. 2012URL http://www.ncpe.ie/wpcontent/uploads/2012/05/Abiraterone-Zytiga-Summary.pdf Accessed 8 November 2018.

30. Zheng HR, Wen F, Wu YF, Wheeler JRC, Li Q. Cost-effectiveness analysis of additional 
docetaxel for metastatic hormone-sensitive prostate cancer treated with androgendeprivation therapy from a Chinese perspective. Eur J Cancer Care (Engl) 2017;26:e12505.

31. Aguiar PN, Barreto CMN, Gutierres B de S, et al. Cost effectiveness of chemohormonal therapy in patients with metastatic hormone-sensitive and non-metastatic high-risk prostate cancer. Einstein (Sao Paulo) 2017;15:349-54.

32. Collins R, Fenwick E, Trowman R, et al. A systematic review and economic model of the clinical effectiveness and cost-effectiveness of docetaxel in combination with prednisone or prednisolone for the treatment of hormone-refractory metastatic prostate cancer. Health Technol Assess 2007;11:iii-iv, xv-xviii, 1-179.

33. Zhong L, Pon V, Srinivas S, et al. Therapeutic Options in Docetaxel-Refractory Metastatic Castration-Resistant Prostate Cancer: A Cost-Effectiveness Analysis. PLoS One 2013;8:e64275.

34. Gong CL, Hay JW. Cost-effectiveness analysis of abiraterone and sipuleucel-T in asymptomatic metastatic castration-resistant prostate cancer. J Natl Compr Canc Netw 2014;12:1417-25.

35. Wilson L, Tang J, Zhong L, et al. New therapeutic options in metastatic castrationresistant prostate cancer: Can cost-effectiveness analysis help in treatment decisions? $J$ Oncol Pharm Pract 2014;20:417-25.

36. Fizazi K, Tran N, Fein L, et al. Abiraterone plus Prednisone in Metastatic, CastrationSensitive Prostate Cancer. N Engl J Med 2017;377:352-60.

37. Wallis CJD, Klaassen Z, Bhindi B, et al. Comparison of Abiraterone Acetate and Docetaxel with Androgen Deprivation Therapy in High-risk and Metastatic Hormonenaïve Prostate Cancer: A Systematic Review and Network Meta-analysis. Eur Urol 2018;73:834-44. 


\section{Figures and Tables}

Fig. 1. Model diagram demonstrating health states. CRPC: castration-resistant prostate cancer; HSPC: hormone-sensitive prostate cancer.

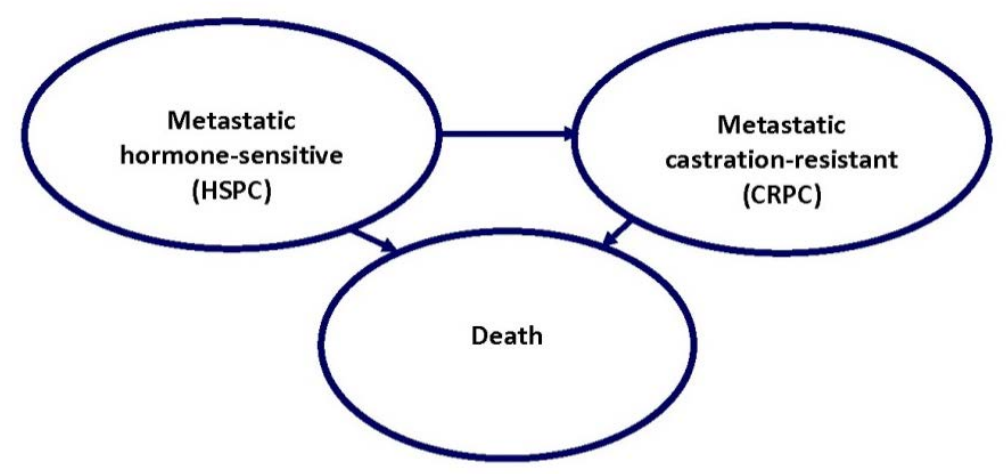

Fig. 2. Model outcomes compared to digitized Kaplan-Meier (KM) data for (A) survival in the metastatic HSPC state; and (B) OS in base case analysis. ADT: androgen-deprivation therapy; CRPC: castration-resistant prostate cancer; D: docetaxel; HSPC: hormone-sensitive prostate cancer; OS: overall survival.

A

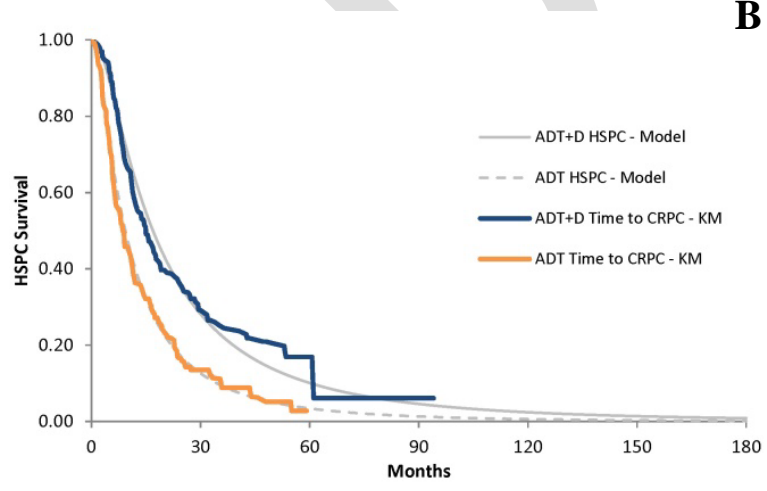

B

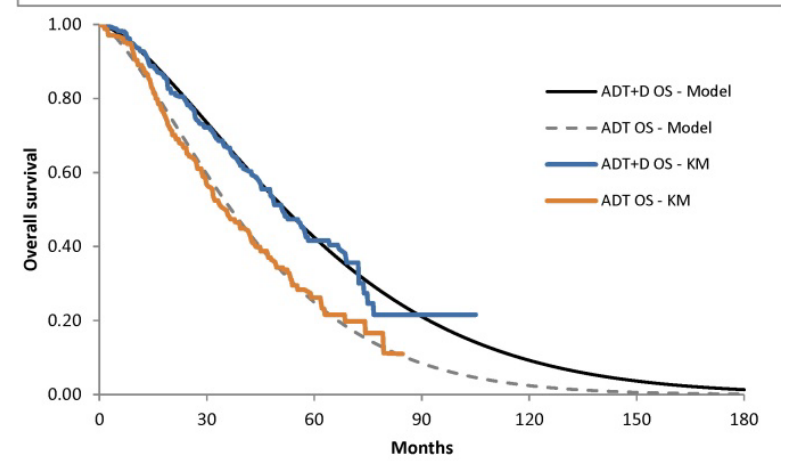


Fig. 3. Results of 5000 Monte Carlo simulations for ADT+D vs. ADT alone to treat patients with high-volume metastatic HSPC presented on (A) cost-effectiveness plane; and (B) costeffectiveness acceptability curve. Willingness-to-pay thresholds of \$50 000/QALY gained and $\$ 100$ 000/QALY gained are also displayed on the cost-effectiveneess plane for reference. ADT: androgen-deprivation therapy; D: docetaxel; HSPC: hormone-sensitive prostate cancer; QALY: quality-adjusted life year.
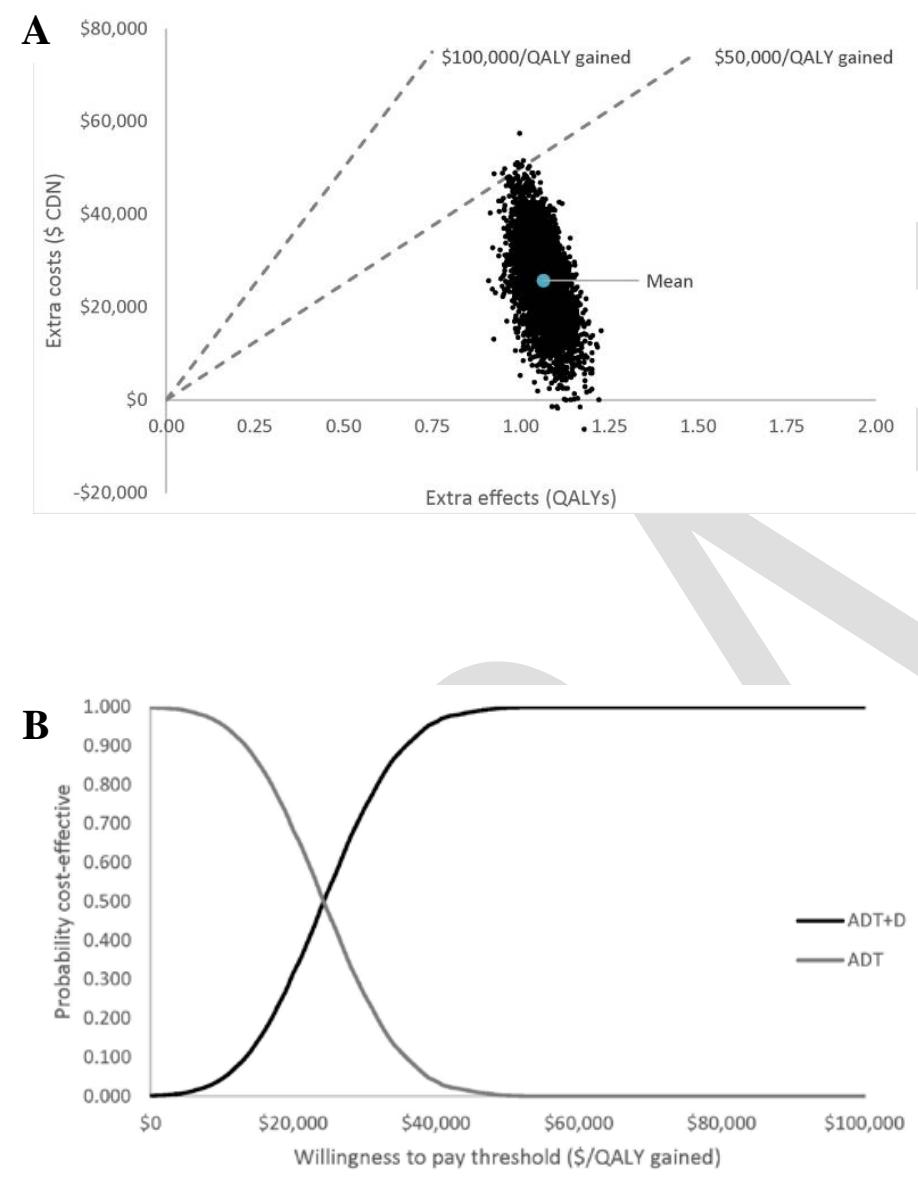
Fig. 4. Tornado diagram for one-way sensitivity and scenario analyses for results of ADT+D vs. ADT alone to treat metastatic HSPC. ADT: androgen-deprivation therapy; CRPC: castrationresistant prostate cancer; D: docetaxel; FN: febrile neutropenia; HSPC: hormone-sensitive prostate cancer; OS: overall survival.
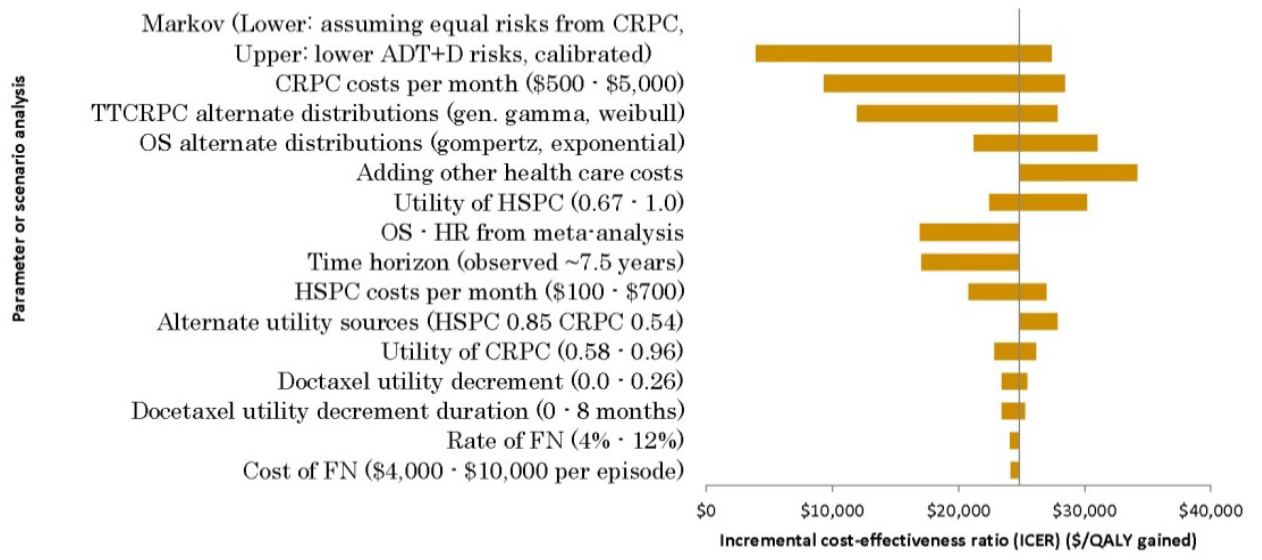

\begin{tabular}{|c|c|c|c|}
\hline & ADT+D & $\begin{array}{l}\text { ADT } \\
\text { alone }\end{array}$ & Source \\
\hline \multicolumn{4}{|l|}{ Survival distributions } \\
\hline Time-to CRPC & $\begin{array}{c}\text { Lognormal } \\
\begin{array}{c}\mu=2.827 \\
\sigma=0.992\end{array}\end{array}$ & $\begin{array}{c}\text { Lognormal } \\
\mu=2.231 \\
\sigma=1.023\end{array}$ & $\begin{array}{l}\text { Based on CHAARTED, Guyot et al } \\
2012^{11,12,19}\end{array}$ \\
\hline OS & $\begin{array}{l}\text { Weibull } \\
\lambda=0.015 \\
\gamma=1.474\end{array}$ & $\begin{array}{l}\text { Weibull } \\
\lambda=0.021 \\
\gamma=1.421\end{array}$ & $\begin{array}{l}\text { Based on CHAARTED, Guyot et al } \\
2012^{11,12,19}\end{array}$ \\
\hline $\begin{array}{l}\text { Sensitivity analysis: OS } \\
\text { hazard ratio for high- } \\
\text { volume disease }\end{array}$ & \multicolumn{2}{|c|}{$\begin{array}{c}0.67 \text { (95\% CI } 0.51- \\
0.88)\end{array}$} & Tucci et al $2016^{14}$ \\
\hline \multicolumn{4}{|l|}{ Probabilities } \\
\hline Febrile neutropenia risk & $8 \%$ & $0 \%$ & Sweeney et al $2015^{10}$ \\
\hline $\begin{array}{l}\text { Sensitivity analysis: } \\
\text { Markov model - } \\
\text { metastatic HSPC to CRPC } \\
\text { or death }\end{array}$ & $\begin{array}{c}\text { Lognormal } \\
\mu=2.827 \\
\sigma=0.992\end{array}$ & $\begin{array}{c}\text { Lognormal } \\
\mu=2.231 \\
\sigma=1.023\end{array}$ & $\begin{array}{l}\text { Based on CHAARTED, Guyot et al } \\
2012^{11,12,19}\end{array}$ \\
\hline $\begin{array}{l}\text { Sensitivity analysis: } \\
\text { Markov model - CRPC to }\end{array}$ & 0.027 & 0.035 & $\begin{array}{l}\text { Calculated based on calibration to } \\
\text { median OS from CHAARTED }{ }^{11,12}\end{array}$ \\
\hline
\end{tabular}


Docetaxel in hormone-sensitive metastatic prostate cancer

\begin{tabular}{|c|c|c|c|}
\hline death & & & \\
\hline $\begin{array}{l}\text { Sensitivity analysis: } \\
\text { Markov model - HSPC to } \\
\text { death }\end{array}$ & \multicolumn{2}{|c|}{ Age-related mortality } & Statistics Canada life tables ${ }^{21}$ \\
\hline \multicolumn{4}{|l|}{ Costs } \\
\hline $\begin{array}{l}\text { Docetaxel monthly cost } \\
(\$ 0.54 / \mathrm{mg})\end{array}$ & $\$ 103$ & $\mathrm{n} / \mathrm{a}$ & Cancer Care Ontario \\
\hline $\begin{array}{l}\text { ADT monthly cost (based } \\
\text { on leuprolide } 22.5 \mathrm{mg} \\
\text { every } 3 \text { months) }\end{array}$ & $\$ 357$ & $\$ 357$ & ODB formulary $^{22}$ \\
\hline $\begin{array}{l}\text { CRPC therapies (based on } \\
\text { abirateratone/enzalutamide } \\
\text { monthly) }\end{array}$ & $\$ 3602$ & $\$ 3602$ & pCODR Economic Guidance Report ${ }^{23}$ \\
\hline $\begin{array}{l}\text { Monthly monitoring and } \\
\text { administration for non-IV } \\
\text { therapy (ADT, } \\
\text { abiraterone/enzalutamide) }\end{array}$ & $\$ 92$ & $\$ 92$ & Cancer Care Ontario costing \\
\hline $\begin{array}{l}\text { Monthly monitoring and } \\
\text { administration cost for IV } \\
\text { therapy (docetaxel) }\end{array}$ & $\$ 455$ & $\mathrm{n} / \mathrm{a}$ & Cancer Care Ontario costing \\
\hline FN hospitalization & $\$ 7326$ & $\mathrm{n} / \mathrm{a}$ & $\begin{array}{l}\text { Lathia et al } 2010^{24} \text {, Statistics Canada } \\
\text { CPI }{ }^{25}\end{array}$ \\
\hline \multicolumn{4}{|l|}{ Utilities } \\
\hline Metastatic HSPC & 0.90 & 0.90 & Bayoumi et al $2000^{26}$ \\
\hline Metastatic CRPC & 0.77 & 0.77 & $\begin{array}{l}\text { National Centre for Pharmacoeconomics } \\
2012^{29}\end{array}$ \\
\hline Disutility for docetaxel & -0.13 & - & Collins et al $2007^{32}$ \\
\hline
\end{tabular}

ADT: androgen-deprivation therapy CI: confidence interval; CRPC: castration-resistant prostate cancer; D: docetaxel; FN: febrile neutropenia; HSPC: hormone-sensitive prostate cancer; IV: intravenous; OS: overall survival. 


\begin{tabular}{|c|c|c|c|}
\hline & ADT D & ADT alone & Incremental \\
\hline Costs & $\$ 140183$ & $\$ 114426$ & $\$ 25757$ \\
\hline HSPC & $\$ 14524$ & $\$ 6873$ & $\$ 7651$ \\
\hline CRPC & $\$ 125659$ & $\$ 107552$ & $\$ 18106$ \\
\hline Life years & 4.767 & 3.489 & 1.278 \\
\hline HSPC & 2.181 & 1.276 & 0.905 \\
\hline CRPC & 2.585 & 2.213 & 0.373 \\
\hline Quality-adjusted life years (QALYs) & 3.915 & 2.852 & 1.063 \\
\hline HSPC & 1.925 & 1.148 & 0.776 \\
\hline CRPC & 1.990 & 1.704 & 0.287 \\
\hline \multicolumn{3}{|c|}{ ICER = Incremental cost/incremental LYs gained } & $\$ 20154$ \\
\hline \multicolumn{3}{|c|}{ ICER = Incremental cost/incremental QALYs gained } & $\$ 24226$ \\
\hline
\end{tabular}

ADT: androgen-deprivation therapy; CRPC: castration-resistant prostate cancer; D: docetaxel; HSP: hormone-sensitive prostate cancer; ICER: Incremental cost-effectiveness ratio. 